\title{
Depot-medication compliance for patients with psychotic disorders: the importance of illness insight and treatment motivation
}

This article was published in the following Dove Press journal:

Neuropsychiatric Disease and Treatment

3 February 2016

Number of times this article has been viewed

\author{
Ernst L Noordraven ${ }^{1,2}$ \\ André I Wierdsma ${ }^{2}$ \\ Peter Blanken ${ }^{3}$ \\ Anthony F T Bloemendaal' \\ Cornelis L Mulder ${ }^{2,4}$ \\ 'Dual Diagnosis Centre (CDP), \\ Parnassia Psychiatric Institute, the \\ Hague, ${ }^{2}$ Department of Psychiatry, \\ Erasmus University Medical Center, \\ Rotterdam, ${ }^{3}$ Parnassia Addiction \\ Research Centre (PARC), Parnassia \\ Psychiatric Institute, the Hague, \\ ${ }^{4}$ Bavo-Europoort Mental Health \\ Care, Parnassia Psychiatric Institute, \\ Rotterdam, the Netherlands
}

Background: Noncompliance is a major problem for patients with a psychotic disorder. Two important risk factors for noncompliance that have a severe negative impact on treatment outcomes are impaired illness insight and lack of motivation. Our cross-sectional study explored how they are related to each other and their compliance with depot medication.

Methods: Interviews were conducted in 169 outpatients with a psychotic disorder taking depot medication. Four patient groups were defined based on low or high illness insight and on low or high motivation. The associations between depot-medication compliance, motivation, and insight were illustrated using generalized linear models.

Results: Generalized linear model showed a significant interaction effect between motivation and insight. Patients with poor insight and high motivation for treatment were more compliant (94\%) (95\% confidence interval [CI]: 1.821, 3.489) with their depot medication than patients with poor insight and low motivation (61\%) (95\% CI: 0.288, 0.615). Patients with both insight and high motivation for treatment were less compliant (73\%) (95\% CI: $0.719,1.315)$ than those with poor insight and high motivation.

Conclusion: Motivation for treatment was more strongly associated with depot-medication compliance than with illness insight. Being motivated to take medication, whether to get better or for other reasons, may be a more important factor than having illness insight in terms of improving depot-medication compliance. Possible implications for clinical practice are discussed.

Keywords: noncompliance, psychotic disorder, illness insight, motivation, depot medication, schizophrenia

\section{Introduction}

Nonadherence to antipsychotic medication has been shown to be a major obstacle to achieving successful treatment outcomes in patients with psychotic disorders. ${ }^{1}$ Approximately $60 \%$ of these patients have difficulty in being adherent over time, leading to problems such as inconsistent symptom control, more relapses, more hospitalizations, and more suicide attempts. ${ }^{2,3}$ Patient, treatment, and environment-related risk factors have all been associated with nonadherence. ${ }^{4}$ This article focuses on two important patient risk factors that appear to have a great impact on whether patients adhere to their prescribed medication regimen: illness insight and motivation for treatment.

Illness insight has been defined by David ${ }^{5}$ as 1 ) the recognition that one has a mental illness, 2) the recognition of the need for treatment, and 3) the ability to relabel unusual mental events as pathological. Of patients with a psychotic disorder, approximately $50 \%$ to $75 \%$ have only a limited degree of illness insight, which often results in poorer treatment outcomes. ${ }^{6-8}$
Correspondence: Ernst L Noordraven Erasmus Medical Center, Psychiatry Department, Gravendijkwal 230, 3015 CE Rotterdam, the Netherlands Tel +3I 107033227

Email e.noordraven@erasmusmc.nl 
The relationship between insight and medication adherence is somewhat ambiguous: ${ }^{9}$ while some studies showed a clear positive association, ${ }^{10-12}$ others either failed to find an association or showed that the effect of insight on medication adherence diminished over time. ${ }^{13,14}$ In sum, illness insight is not enough to improve medication compliance. Neither is it a prerequisite for taking medication. Perhaps patients with poor insight who accept their medication are simply motivated to take medication, whether or not they completely understand or acknowledge their mental illness.

Motivation has been defined as "the probability that a person will enter into, continue, and adhere to a specific change strategy". ${ }^{15}$ Motivation for treatment among psychotic patients has been studied extensively in therapeutic settings, and is positively associated with effective treatment outcomes. ${ }^{16}$ Often, however, motivation for treatment is lacking in patients with psychotic disorders such as schizophrenia. ${ }^{17}$ As a result, these patients are less willing to adhere to antipsychotic medication prescriptions, to engage in treatment activities, or to show up for appointments. Neither are they likely to benefit from their treatment. As a negative symptom of schizophrenia disorder, lack of motivation is a common problem that is difficult for patients and clinicians to address.

To increase the benefits of treatment, patients can be prescribed antipsychotic depot medication, which is easier to monitor, has long-lasting effects, and reduces relapse rates more than oral medication. ${ }^{18}$ However, since the effects of depot medication in different treatment settings are somewhat unclear, ${ }^{19}$ we decided to focus on the effects of antipsychotic depot medication among outpatients with a psychotic disorder.

Thus, impaired insight and lack of motivation can have a negative impact on medication compliance. It remains unclear, however, how these risk factors relate to each other and to medication compliance. This is important for developing intervention studies that might focus more on developing illness insight or increasing motivation for treatment. Therefore, in a cross-sectional study we explored the associations between illness insight, motivation for treatment, and adherence to antipsychotic depot medication.

\section{Methods}

\section{Patients}

Baseline data were obtained from 169 patients participating in Money for Medication, an ongoing randomized controlled trial on the effectiveness of financial incentives for improving adherence to depot medication (for protocol details, see Noordraven et $\mathrm{al}^{20}$ ). Patients were recruited from three mental health care institutions in the Netherlands that primarily treat patients with psychotic and other severe mental disorders (often with comorbid substance-use disorder). Patients met the following inclusion criteria: age between 18 and 65 years, having a psychotic disorder, taking antipsychotic depot medication or an indication to start using it, receiving outpatient treatment, and having given written informed consent on participating in a randomized controlled trial. There were two exclusion criteria: inability to participate due to cognitive impairments and inability to participate due to insufficient understanding of the Dutch language. The study was approved by the accredited Dutch Medical Ethical Trial Committee at Erasmus University Medical Center (Trial Registration NTR2350).

\section{Procedure}

Candidate participants were selected from the caseloads on the basis of the inclusion and exclusion criteria. The baseline interview was conducted after written informed consent had been given and before randomization. All interviews were conducted by psychologists who had received professional training in the Positive And Negative Syndrome Scale (PANSS) and Health of the Nation Outcome Scales (HoNOS) interview and its subsequent scoring procedures. Per interview, all participants received a remuneration of $€ 20$. Demographic variables, DSM-IV diagnoses on axis I and II, and psychiatric history were collected during the first interview and from patients' medical records. In the trial, randomization took place after the first interview, whereas for this study all patients were treated as one sample. For a more detailed protocol description, see Noordraven et al. ${ }^{20}$

\section{Measurements}

\section{Medication compliance}

The medication possession ratio (MPR), first reported by Sclar et $\mathrm{al}^{21}$ was used as a measure for depot-medication compliance. MPR is defined as the number of accepted depots of antipsychotic medication divided by the number of depots of antipsychotic medication prescribed, that is, the number of supplies needed for continuous use of antipsychotic medication. Each patient's MPR at baseline was determined on the basis of the 4 months prior to the baseline interview.

\section{Illness insight}

Insight was measured using the Dutch version of the PANSS, ${ }^{22}$ a 30 -item semistructured interview intended to determine the presence of positive and negative symptoms and general psychopathology. Illness insight was assessed on 
the basis of item A12 of the PANSS "Do you have a psychiatric disorder or mental health problem?" in which the patient is also asked to elaborate his answer. Specifically, this item describes a patient's ability to acknowledge his or her psychiatric disorder, need for treatment, and ability to make future plans. Items were scored on a scale from 1 (illness insight present) to 7 (active denial of having a psychiatric disorder). Response scores for insight (item A12) were dichotomized into patients having "poor insight" (scoring 3-7 on the insight item) and "high illness insight" (scoring 1-2).

\section{Motivation for treatment}

Motivation for treatment was assessed on the basis of an item added to the Dutch version of the HoNOS, ${ }^{23}$ which describes a patient's motivation in terms of cooperation, personal interest, and possible resistance to treatment: "How motivated are you for your current treatment?" As with the other items of the HoNOS, this item was rated on a 5-point scale ranging from 0 (no problems) to 4 (very severe problems). Patient scores were dichotomized into "high motivation for treatment" (scoring 0-1 on the motivation item) and "low motivation for treatment" (scoring 2-4).

\section{Missing values}

Due to administrative errors, data for the total sample lacked baseline data for three patients. Similarly, 13 values were missing for compliance rates, 2 for motivation, and 2 for insight. One patient had missing values for both motivation and insight. Therefore, in total, 19 patients were excluded from the analyses. Sensitivity analyses using imputed data on average values did not show different results.

\section{Statistical analyses}

We used generalized linear models to estimate the effect of motivation and insight on the number of depots accepted as a percentage of the number of depots prescribed. Because MPR values are proportion data, we assumed binomial error distribution and used the logit link function. Patients were grouped into four categories: 1) those with low motivation and poor insight $(\mathrm{n}=62) ; 2)$ those with high motivation but poor insight $(\mathrm{n}=17) ; 3)$ those with low motivation but a high degree of insight ( $\mathrm{n}=17)$; and 4 ) those whose motivation and illness insight were high $(n=54)$. All groups were entered as one categorical predictor variable. Since patients with low motivation and poor insight had the lowest compliance rate $(65.8 \%)$, we used this category as our reference group. All statistical analyses were performed using SPSS version 21.0 (IBM Corporation, Armonk, NY, USA).
Table I Baseline characteristics of the study sample $(\mathrm{N}=166)$

\begin{tabular}{|c|c|}
\hline Variable & $\begin{array}{l}\text { Descriptive } \\
\text { statistics }\end{array}$ \\
\hline Age (years), mean (SD) & $40.7(10.2)$ \\
\hline \multicolumn{2}{|l|}{ Sex, N (\%) } \\
\hline Male & $124(74.3)$ \\
\hline Female & $42(25.1)$ \\
\hline Duration of illness (years), mean (SD) & $12.3(8.5)$ \\
\hline \multicolumn{2}{|l|}{ Ethnicity, N (\%) } \\
\hline Dutch & $64(38.3)$ \\
\hline Surinamese & $39(23.4)$ \\
\hline Other & $63(38.3)$ \\
\hline Outpatient commitment measures, $\mathrm{N}(\%)^{*}$ & $60(36.7)$ \\
\hline \multicolumn{2}{|l|}{ Diagnosis, N (\%) } \\
\hline Schizophrenia paranoid type & $96(57.5)$ \\
\hline Schizoaffective disorder & $18(10.8)$ \\
\hline Psychotic disorder NOS & $18(10.8)$ \\
\hline Schizophrenia disorganized type & II (6.6) \\
\hline Other schizophrenic disorders & $24(14.3)$ \\
\hline \multicolumn{2}{|l|}{ Medication at baseline, $\mathrm{N}(\%)$} \\
\hline First-generation antipsychotics & $125(75.8)$ \\
\hline Second-generation antipsychotics & $39(23.6)$ \\
\hline Depot medication & $140(82.6)$ \\
\hline Motivation, median (interquartile range) & $2(0-2)$ \\
\hline Illness insight, median (interquartile range) & $3(1-4)$ \\
\hline No of psych admissions, ${ }^{* *}$ median (interquartile range) & $I(0-3)$ \\
\hline No of admission days, $* *$ median (interquartile range) & $44(0-120)$ \\
\hline
\end{tabular}

Notes: *Mental health law for compulsory admissions and outpatient commitment in the Netherlands. **Three years preceding the baseline interview.

Abbreviations: SD, standard deviation; NOS, not otherwise specified.

\section{Results}

\section{Patient characteristics}

Table 1 presents the sociodemographic and clinical data of all patients at baseline. Data for motivation, insight, and medication compliance at baseline are presented in Table 2 .

\section{Relationship between insight and compliance}

Insight was positively associated with MPR $(\beta=1.471$, 95\% confidence interval $[\mathrm{CI}]:[0.468,1.00], P<0.001)$, (Table 2). Patients with high illness insight were almost $10 \%$

Table 2 Mean medication possession ratio scores at baseline interview by motivation and insight

\begin{tabular}{lll}
\hline Variable & $\begin{array}{l}\text { Medication } \\
\text { possession ratio (\%) }\end{array}$ & $\mathbf{N}$ \\
\hline Insight & & \\
Poor & 71.9 & 79 \\
High & 81.6 & 71 \\
Motivation & & \\
Low & 68.0 & 79 \\
High & 85.8 & 71 \\
\hline
\end{tabular}


more compliant with their prescribed antipsychotic depot medication than patients with poor insight.

\section{Relationship between motivation and compliance}

Motivation, too, was positively associated with MPR ( $\beta=1.133,95 \%$ CI: $[0.854,1.413], P<0.001$ ), (Table 2 ). Patients with a high motivation for treatment were $18 \%$ more compliant with the antipsychotic depot medication prescribed to them than patients with low motivation for treatment.

\section{Insight, motivation, and their association with compliance}

Our results showed a significant interaction effect between motivation and insight (Figure 1). Patients with poor insight but high motivation for treatment were more compliant with their medication (94\%; $\beta=2.655,95 \%$ CI: [1.821, 3.489], $P<0.001)$ than patients with poor insight and low motivation (61\%; $\beta=1.570,95 \%$ CI: [0.288, 0.615], $P<0.001)$. Patients with high insight and motivation $(73 \% ; \beta=1.017,95 \% \mathrm{CI}$ : $[0.719,1.315], P<0.001)$ and patients with high insight but low motivation (74\%, $\beta=1.028,95 \%$ CI: [0.547, 1.510], $P<0.001)$ were more compliant than patients with low motivation and poor insight $(61 \%)$. Switching our reference category to patients with high motivation and insight (73\%) allowed us to compare their medication compliance with patients with high insight and low motivation (74\%), which showed no significant difference. However, patients with high motivation and poor insight (94\%) were more compliant than those with high insight. This suggests that insight is not only less strongly associated with compliance than motivation, but also that the level of compliance in patients with high motivation may be reduced by insight.

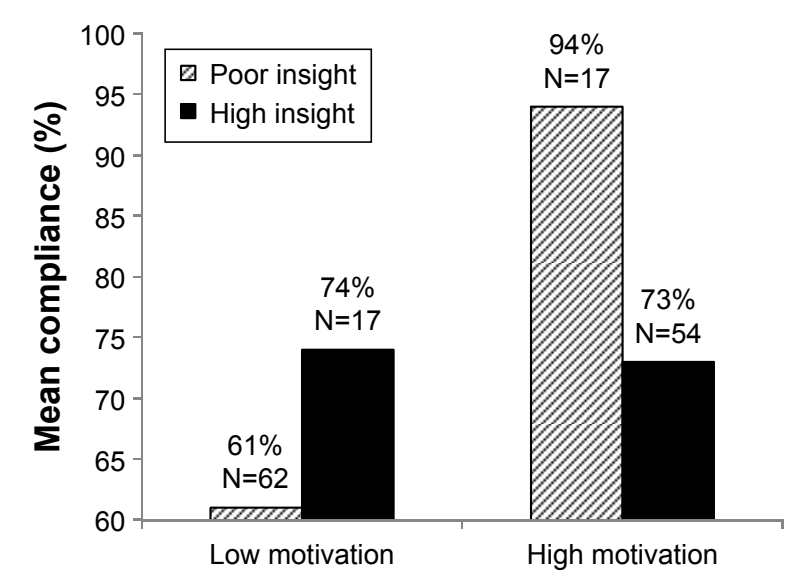

Figure I Interaction effect between patient categories.

\section{Discussion}

The main finding of our study was that motivation for treatment seems to be more important than illness insight for compliance with depot medication in psychotic disorder patients. Specifically, we found that patients with a high motivation for treatment were most compliant with their medication, independent of insight. Unexpectedly, patients with high insight and high motivation showed less compliance as compared to patients with poor insight and high motivation.

It may seem contradictory that patients with poor illness insight were willing to accept their antipsychotic depot medication. From this perspective, one could argue that having illness insight is not a prerequisite for achieving compliance. In other words, it may be more important to be motivated to take medication - whether to get better, or for other reasons - than to have illness insight with regard to improving depot-medication compliance.

Our findings may have two important clinical consequences for increasing compliance with depot medication. First, we found that patients with poor insight who were highly motivated for treatment had over 30\% more compliance. Therefore, it may be more important in patients with poor insight to improve motivation for treatment as a first step, for example, by using motivational interviewing strategies $^{24}$, instead of immediately trying to enhance illness insight.

Second, when addressing illness insight, we have to be aware of possible negative consequences regarding compliance, since we found that a high degree of illness insight in highly motivated patients was accompanied by a level of compliance that was $\sim 20 \%$ lower than that in patients with poor insight. This suggests that greater illness insight is not necessarily associated with higher compliance rates in patients who are highly motivated, but may sometimes be accompanied by lower medication acceptance. This is surprising, since it has always been assumed that it would be better to have good insight. Although our finding that greater illness insight does not lead to better compliance is counterintuitive, it may be that patients who are highly motivated and also have illness insight want to use other treatment options, such as oral medication and/or psychotherapy.

\section{Limitations}

Three important limitations of this study should be considered. First, our cross-sectional design makes it difficult to infer causality. ${ }^{25}$ Longitudinal and experimental studies are 
needed to further study the associations between motivation, illness insight, and compliance.

The second limitation is that motivation and insight were both measured using a single item. In previous studies, however, the PANSS insight item showed high correlations with questionnaires assessing insight such as the Insight and Treatment Attitudes Questionnaire or the Schedule for the Assessment of Insight. ${ }^{26,27}$ With respect to the HoNOS motivation item, earlier studies also found high and significant correlations with scales measuring the motivation construct, including the Treatment Entry Questionnaire and the Treatment Motivation Scale..$^{28}$

The third limitation is that only patients on depot medication participated in this study. Associations between illness insight and motivation may be different for patients on daily oral medication.

\section{Conclusion}

Motivation and illness insight both play an important role in a patient's acceptance of antipsychotic depots. Attempts to improve compliance should be made carefully, first by assessing their level of motivation and illness insight. The clinician can then make an informed decision on the extent of any interventions intended to improve compliance through improved motivation and/or insight. Our results suggest that it may be more important to improve compliance by improving motivation than by enhancing insight, especially in patients whose insight is already poor. It will be interesting to investigate whether this is also the case with compliance with daily oral antipsychotic medications. Ultimately, different treatment protocols might be used for patients with different combinations of insight and motivation. In everyday practice, it may be best for clinicians not to focus on either insight or motivation, but to recognize that the most important factor in understanding compliance with antipsychotic medication lies in the combination of the two. Future research should establish whether these associations are valid over time and which interventions clinicians should use to improve motivation and illness insight among patients with a psychotic disorder.

\section{Disclosure}

The authors report no conflicts of interest in this work.

\section{References}

1. Gray R, Leese M, Bindman J, et al. Adherence therapy for people with schizophrenia European multicentre randomised controlled trial. Br J Psychiatry. 2006;189(6):508-514.
2. Llorca PM. Partial compliance in schizophrenia and the impact on patient outcomes. Psychiatry Res. 2008;161(2):235-247.

3. Weiden PJ, Kozma C, Grogg A, Locklear J. Partial compliance and risk of rehospitalization among California medicaid patients with schizophrenia. Psychiatr Serv. 2004;55(8):886-891.

4. Higashi K, Medic G, Littlewood KJ, Diez T, Granström O, De Hert M. Medication adherence in schizophrenia: factors influencing adherence and consequences of nonadherence, a systematic literature review. Ther Adv Psychopharmacol. 2013;3(4):200-218.

5. David AS. Insight and psychosis. Br J Psychiatry. 1990;156(6): 798-808.

6. Buchy L, Torres IJ, Liddle PF, Woodward TS. Symptomatic determinants of insight in schizophrenia spectrum disorders. Compr Psychiatry. 2009;50(6):578-583.

7. Quee PJ, van der Meer L, Bruggeman R, et al. Insight in psychosis: relationship with neurocognition, social cognition and clinical symptoms depends on phase of illness. Schizophr Bull. 2011;37(1):29-37.

8. Velligan DI, Weiden PJ, Sajatovic M, et al. The expert consensus guideline series: adherence problems in patients with serious and persistent mental illness. J Clin Psychiatry. 2009;70(4):1-46.

9. Lincoln TM, Lüllmann E, Rief W. Correlates and long-term consequences of poor insight in patients with schizophrenia. A systematic review. Schizophr Bull. 2007;33(6):1324-1342.

10. Acosta FJ, Bosch E, Sarmiento G, Juanes N, Caballero-Hidalgo A, Mayans T. Evaluation of noncompliance in schizophrenia patients using electronic monitoring (MEMS) and its relationship to sociodemographic, clinical and psychopathological variables. Schizophr Res. 2009;107(2-3):213-217.

11. Kozuki Y, Froelicher ES. Lack of awareness and nonadherence in schizophrenia. West J Nurs Res. 2003;25(1):57-74.

12. Perkins DO, Gu H, Weiden PJ, McEvoy JP, Hamer RM, Lieberman JA. Predictors of treatment discontinuation and medication nonadherence in patients recovering from a first episode of schizophrenia, schizophreniform disorder, or schizoaffective disorder: a randomized, double-blind, flexible-dose, multicenter study. J Clin Psychiatry. 2008;69(1):106-113.

13. McEvoy JP, Freter S, Everett G, et al. Insight and the clinical outcome of schizophrenic patients. J Nerv Ment Dis. 1989;177(1):48-51.

14. Yen CF, Chen CS, Ko CH, et al. Relationships between insight and medication adherence in outpatients with schizophrenia and bipolar disorder: prospective study. Psychiatry Clin Neurosci. 2005;59(4): 403-409.

15. Miller WR, Rollnick S. Motivational Interviewing: Preparing People to Change Addictive Behavior. New York: Guilford Press; 1991:1-300.

16. Yamada AM, Lee KK, Dinh TQ, Barrio C, Brekke JS. Intrinsic motivation as a mediator of relationships between symptoms and functioning among individuals with schizophrenia spectrum disorders in a diverse urban community. J Nerv Ment Dis. 2010;198(1):28-34.

17. Choi J, Medalia A. Intrinsic motivation and learning in a schizophrenia spectrum sample. Schizophr Res. 2011;118(1-3):12-19.

18. Novick D, Haro JM, Bertsch J, Anand H, Jemiai N, Haddad PM. Comparison of treatment discontinuation and hospitalization among nonadherent patients initiating depot or oral typical antipsychotic medications. Int Clin Psychopharmacol. 2012;27(5):275-282.

19. Leucht C, Heres S, Kane JM, Kissling W, Davis JM, Leucht S. Oral versus depot antipsychotic drugs for schizophrenia - a critical systematic review and meta-analysis of randomised long-term trials. Schizophr Res. 2011;127(1-3):83-92.

20. Noordraven EL, Audier CH, Staring AB, et al. Money for medication: a randomized controlled study on the effectiveness of financial incentives to improve medication adherence in patients with psychotic disorders. BMC Psychiatry. 2014;14(1):343.

21. Sclar DA, Chin A, Skaer TL, Okamato MP, Nakahiro RK, Gill MA. Effect of health education in promoting prescription refill compliance among patients with hypertension. Clin Ther. 1991;13(4):489-495. 
22. Kay SR, Fiszbein A, Opler LA. The Positive and Negative Syndrome Scale (PANSS) for schizophrenia. Schizophr Bull. 1987;13(2): 261-272.

23. Kortrijk HE, Staring ABP, Van Baars AW, Mulder CL. Involuntary admission may support treatment outcome and motivation in patients receiving assertive community treatment. Soc Psychiatry Psychiatr Epidemiol. 2010;45:245-252.

24. Barkhof E, Meijer CJ, de Sonneville LM, Linszen DH, de Haan L. The effect of motivational interviewing on medication adherence and hospitalization rates in nonadherent patients with multi-episode schizophrenia. Schizophr Bull. 2013;39(6):1242-1251.

25. Flanders WD, Lin L, Pirkle JL, Caudill SP. Assessing the direction of causality in cross-sectional studies. Am J Epidemiol. 1992;135(8): 926-935.
26. Jørgensen R, Licht RW, Lysaker PH, et al. Effects on cognitive and clinical insight with the use of guided self-determination in outpatients with schizophrenia: a randomized open trial. Eur Psychiatry. 2015;30(5): 655-663.

27. Sanz M, Constable G, Lopez-Ibor I, Kemp R, David AS. A comparative study of insight scales and their relationship to psychopathological and clinical variables. Psychol Med. 1998;28:437-446.

28. Mulder CL, Jochems E, Kortrijk HE. The motivation paradox: higher psychosocial problem levels in severely mentally ill patients are associated with less motivation for treatment. Soc Psychiatry Psychiatr Epidemiol. 2014;49(4):541-548.

\section{Publish your work in this journal}

Neuropsychiatric Disease and Treatment is an international, peerreviewed journal of clinical therapeutics and pharmacology focusing on concise rapid reporting of clinical or pre-clinical studies on a range of neuropsychiatric and neurological disorders. This journal is indexed on PubMed Central, the 'PsycINFO' database and CAS, and is the official journal of The International Neuropsychiatric Association (INA). The manuscript management system is completely online and includes a very quick and fair peer-review system, which is all easy to use. Visit http://www.dovepress.com/testimonials.php to read real quotes from published authors.

Submit your manuscript here: http://www.dovepress.com/neuropsychiatric-disease-and-treatment-journal 